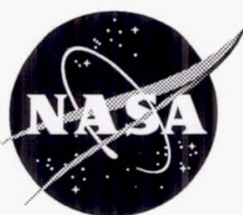

Wind Code Application to External Forebody Flowfields With Comparisons to Experimental Results

F.C. Frate

Dynacs Engineering Company, Inc., Brook Park, Ohio

H.D. Kim

Glenn Research Center, Cleveland, Ohio 
Since its founding, NASA has been dedicated to the advancement of aeronautics and space science. The NASA Scientific and Technical Information (STI) Program Office plays a key part in helping NASA maintain this important role.

The NASA STI Program Office is operated by Langley Research Center, the Lead Center for NASA's scientific and technical information. The NASA STI Program Office provides access to the NASA STI Database, the largest collection of aeronautical and space science STI in the world. The Program Office is also NASA's institutional mechanism for disseminating the results of its research and development activities. These results are published by NASA in the NASA STI Report Series, which includes the following report types:

- TECHNICAL PUBLICATION. Reports of completed research or a major significant phase of research that present the results of NASA programs and include extensive data or theoretical analysis. Includes compilations of significant scientific and technical data and information deemed to be of continuing reference value. NASA's counterpart of peerreviewed formal professional papers but has less stringent limitations on manuscript length and extent of graphic presentations.

- TECHNICAL MEMORANDUM. Scientific and technical findings that are preliminary or of specialized interest, e.g., quick release reports, working papers, and bibliographies that contain minimal annotation. Does not contain extensive analysis.

- CONTRACTOR REPORT. Scientific and technical findings by NASA-sponsored contractors and grantees.
- CONFERENCE PUBLICATION. Collected papers from scientific and technical conferences, symposia, seminars, or other meetings sponsored or cosponsored by NASA.

- SPECIAL PUBLICATION. Scientific, technical, or historical information from NASA programs, projects, and missions, often concerned with subjects having substantial public interest.

- TECHNICAL TRANSLATION. Englishlanguage translations of foreign scientific and technical material pertinent to NASA's mission.

Specialized services that complement the STI Program Office's diverse offerings include creating custom thesauri, building customized data bases, organizing and publishing research results ... even providing videos.

For more information about the NASA STI Program Office, see the following:

- Access the NASA STI Program Home Page at http://www.sti.nasa.gov

- E-mail your question via the Internet to help@sti.nasa.gov

- Fax your question to the NASA Access Help Desk at 301-621-0134

- Telephone the NASA Access Help Desk at 301-621-0390

- Write to: NASA Access Help Desk NASA Center for AeroSpace Information 7121 Standard Drive Hanover, MD 21076 


\section{Wind Code Application to External Forebody Flowfields With Comparisons to Experimental Results}

F.C. Frate

Dynacs Engineering Company, Inc., Brook Park, Ohio

H.D. Kim

Glenn Research Center, Cleveland, Ohio

Prepared for the 39th Aerospace Sciences Meeting and Exhibit sponsored by the American Institute of Aeronautics and Astronautics Reno, Nevada, January 8-11, 2001

National Aeronautics and Space Administration

Glenn Research Center 


\section{Acknowledgments}

The authors would like to thank J. Slater for assembling the grids for this study. T. Bencic is also acknowledged for working with the TSP system and supplying the relevant data.

Available from

NASA Center for Aerospace Information 7121 Standard Drive

Hanover, MD 21076
National Technical Information Service 5285 Port Royal Road Springfield, VA 22100

Available electronically at http://gltrs.grc.nasa.gov/GLTRS 


\title{
WIND CODE APPLICATION TO EXTERNAL FOREBODY FLOWFIELDS WITH COMPARISONS TO EXPERIMENTAL RESULTS
}

\author{
F.C. Frate \\ Dynacs Engineering Company, Inc. \\ Brook Park, Ohio 44142 \\ H.D. Kim \\ National Aeronautics and Space Administration \\ Glenn Research Center \\ Cleveland, Ohio 44135
}

\begin{abstract}
The WIND Code, a general purpose Navier-Stokes solver, has been utilized to obtain supersonic external flowfield CFD solutions over an axisymmetric, parabolic forebody with comparisons made to wind tunnel experimental results. Various cases have been investigated at supersonic freestream conditions ranging from Mach 2.0 to 3.5 , at $0^{\circ}$ and $3^{\circ}$ angles-of-attack, and with either a sharp-nose or blunt-nose forebody configuration. Both a turbulent (Baldwin-Lomax algebraic turbulence model) and a laminar model have been implemented in the CFD. Obtaining the solutions involved utilizing either the parabolized- or full-NavierStokes analyses supplied in WIND. Comparisons have been made with static pressure measurements, with boundary-layer rake and flowfield rake pitot pressure measurements, and with temperature-sensitive paint experimental results. Using WIND's parabolized NavierStokes capability, grid sequencing, and the BaldwinLomax algebraic turbulence model allowed for significant reductions in computational time while still providing good agreement with experiment. Given that CFD and experiment compare well, WIND is found to be a good computational platform for solving this type of forebody problem, and the grids developed in conjunction with it will be used in the future to investigate varying freestream conditions not tested experimentally.
\end{abstract}

\section{Introduction}

A reusable, Rocket Based Combined Cycle (RBCC), single-stage-to-orbit launch vehicle concept called GTX (formerly known as Trailblazer) ${ }^{1}$ is one that will use air-breathing propulsion to aid in reducing the required propellant mass fraction and, therefore, cost of access to space. Figure 1(a) shows a scaled-down model of the vehicle concept that was tested in NASA Glenn's 10-by-10 foot Supersonic Wind Tunnel (SWT) with Mach numbers ranging from 2.0 to 3.5 and at various angles-of-attack to experimentally evaluate the external aerodynamics and mass capture characteristics. ${ }^{2}$ It was desired to model the experimental set-up (as far as the forebody is concerned) and compute the external flowfield to a reasonably accurate extent with CFD. This was done in order to develop a capability that could supply any additional external flowfield information (which may eventually affect the inlets) not supplied by the experiment. As part of the project scope, the use of grid sequencing and a parabolized Navier-Stokes $^{3}$ (PNS) analysis was investigated in order to save computational time and yet still obtain high fidelity CFD results. A "simultaneous" integration between experimental and CFD efforts was made possible because of these capabilities.

\section{Experiment}

\section{Methods And Procedures}

The forebody hardware had the following parabolic contour: $r=-0.00116105 x^{2}+0.223959 x$, where $r=$ radius and $\mathrm{x}=$ axial distance. Either a sharp-nose tip or a 0.464 in. radius blunt-nose tip was used during each test. This parabolic geometry transitioned smoothly into a constant radius cylindrical section from $x=96.45$ in. and aft (referenced from the sharp-nose forebody tip). The conditions investigated for the sharp- and blunt-nose forebodies included Mach 2.0, 2.5, 3.0 and 3.5, with a unit Reynolds Number (Re\#/ft) of $\sim 3.3 \times 10^{6}, 2.7 \times 10^{6}$, $2.5 \times 10^{6}$ and $2.5 \times 10^{6}$, respectively. Angles-of-attack $(\alpha)$ equal to $0^{\circ}, \pm 3^{\circ}, \pm 6^{\circ}$ and $\pm 9^{\circ}$ were investigated at each freestream condition, though only $0^{\circ}$ and $3^{\circ}$ are focused on in this paper. All angles are with respect to the model's installation in the 10- by 10 -foot SWT.

During several sharp-nose experimental tests for $\alpha=0^{\circ}$, a grit was applied at $x=3.0 \mathrm{in}$. in order to trip the boundary-layer to turbulent. Sharp-nose forebody experimental results with and without application of the grit will be presented and compared to the turbulent and laminar CFD solutions.

For the sharp-nose configuration, the CFD results can be directly compared with the following experimental data:

1) Static pressure measurements along the forebody,

2) Boundary-layer rake and flowfield rake measurements (both measuring pitot pressures) at various axial and circumferential forebody locations, and

3) Temperature-sensitive paint (TSP) results. 
For the blunt-nose forebody, only the boundary-layer rake pitot pressure measurements were available and could be compared with the CFD solutions.

\section{Computational Model}

Coordinates for the forebody were supplied and incorporated into the gridding process. A rough schematic and general description of what was modeled in the CFD is shown in Fig. 1(b). The experimental data to be used in comparison with the CFD data is relevant up to a point just in front of the three propulsion pods' centerbody tips at $\mathrm{x}=93.33 \mathrm{in}$. (which are visible in Fig. 1(a)). The pods were not modeled in the CFD process. However, the grid did model the constant cylindrical section up to $x=150$ in. Therefore, experimental data obtained circumferentially between the pod positions is only valid (in comparisons with the CFD) at locations where the first effects from each centerbody's conical shock would still not be disturbing the forebody flowfield.

In modeling the sharp-nose forebody configuration, a 3-D $180^{\circ}$ single zone, structured grid with two levels of sequencing was created (modeling $\mathrm{x}=-25$ to 150 in.) using ICEM CFD Mulcad. ${ }^{4}$ The grid is shown in Fig. 2(a). The grid contains 161 grid points axially in the $i$-direction, 149 radially in the $j$-direction, and 61 circumferentially in the $k$-direction. Because of the way the sharp-nose grid was assembled, the PNS capability (for space marching in the axial direction) could be used in the WIND solution process. The wall spacing at the body was 0.0002 in. (based on $y^{+}$ criteria), with 0.1 in. axial spacing at the nose and 1.0 in. axial spacing along the forebody surface. The sharp-nose grid $i=1$ boundary condition (B.C.) was set to a frozen B.C., as was the $j=j_{\max }$ B.C. An outflow B.C. was used for $i=i_{\max }$. The $j=1$ B.C. consisted of a singular axis B.C. ahead of the forebody and a viscous wall B.C. along the forebody. For this $180^{\circ}$ grid, $k=1$ and $k=k_{\max }$ were reflection B.C.'s.

A separate $3-\mathrm{D} 180^{\circ}$ grid was created for the bluntnose forebody configuration, with a 0.464 in. radius nose-tip. The grid created for this blunt-nose forebody can be seen in Fig. 2(b). To accurately grid the forebody in the nose-tip region, a somewhat "polar grid" was created, with the $i=1$ location corresponding to the singular axis boundary condition. The grid wraps around the nose while traveling in the $i$-direction. The grid dimensioning corresponds to $141 \times 149 \times 61$ (for two levels of sequencing). The wall spacing was equal to 0.0002 in., with 0.06 in. axial spacing at the nose, and 1.0 in. axial spacing along the body. An FNS analysis instead of a PNS analysis was the solution method used for the blunt-nose grid because of the singular axis at $i=1$. Depending on processor availability, for certain runs the grid could be split into two zones and run using WIND's parallel processor capability.
The WIND Code's capabilities and documentation are described in detail in Ref. 5. WIND Version 3.0 was used in all CFD calculations. The viscous equations were solved during each run of WIND using either a turbulent (Baldwin-Lomax algebraic model) or a laminar model. WIND was run in standard mode, using the ideal gas model along with the Sutherland law for the transport properties. An adiabatic wall condition was utilized. The parameters specified within the WIND input file included total freestream conditions, a downstream keyword of pressure extrapolate always, specification of the turbulence or laminar model, sequencing specification, and PNS- or FNS-specific commands. WIND was run mainly in single processor mode using either R10000 (225 MHz) or R12000 $(400 \mathrm{MHz})$ SGI processors, depending on availability.

The sharp-nose grid solutions involved running the PNS capability to convergence. In addition to accurately resolving the boundary-layer, the L2 norm of the residuals was seen to drop $\sim 3$ orders of magnitude (except at the nose-tip) to a value usually below $10^{-4}$. The blunt-nose grid (with FNS capability) was investigated for convergence by monitoring the integrated pressure and viscous forces, the boundarylayer profile, the flowfield, and usually a drop of $\sim 3$ orders of magnitude in the L2 norm of the residuals. The primary grid sequencing used corresponded to every other grid point from the grid dimensions quoted above (both for sharp- and blunt-nose cases). This approach was selected based on a grid sequencing/ convergence study (per the methods of Ref. 6) described in the Appendix.

\section{Results And Discussion}

Results from the grid convergence study (see Tables 1 and 2) showed the following general trends with respect to CPU time. The PNS cases (for sharpnose grids) ran 5 to 10 times faster than the FNS cases (blunt-nose grid). Using the Medium grids (every other grid point) instead of the Fine grids reduced the run time by approximately a factor of 25 . Use of the PNS capability where possible, use of the coarser (Medium grid spacing) grids, and use of an algebraic turbulence model (instead of a one- or two-equation model) helped greatly reduce the computational time, given that most of the cases were run on a single processor. This allowed for a greater number of comparisons that could be made with experimental results. These comparisons will now be discussed.

\section{Sharp-nose Results}

Normalized Surface Static Pressure Distributions. Fig. 3(a) displays the normalized static pressure $\left(\mathrm{P}_{\text {static }}\right.$ ' $\left.P_{\text {tunnel static }}\right)$ plotted versus the axial distance from the nose-tip for the Mach 2.5, $\alpha=0^{\circ}$ test case. Turbulent CFD results are plotted with the corresponding experimental (without grit-applied) static pressure data. The 
CFD results are seen to compare well with the experimental data. At $\alpha=0^{\circ}$, it is expected that all static pressures at a given axial station along the circumferential ( $\theta$-direction) should be equal (i.e., no pitch or yaw). The figure shows that, in general, this is the case here. $\theta$ is measured, looking upstream, traveling counter-clockwise (or clockwise because of symmetry) from the top of the model's forebody to the bottom (see Fig. 1(b)). The static pressure is highest at the nose-tip, after which it steadily decreases as the flow expands over the parabolic forebody surface. The slight waviness in the CFD solution near the nose-tip region may be due to the way the PNS solution method handles the coarser grid at this "discontinuity."

For Mach $2.5\left(\alpha=3^{\circ}\right)$, Fig. 3(b) shows CFD and experimental results coincide fairly well. When at angle-of-attack, higher static pressures are expected on the windward side $\left(\theta=180^{\circ}\right.$ line) of the forebody as compared to the $120^{\circ} / 240^{\circ}$ lines, the $90^{\circ} / 270^{\circ}$ lines and the leeward side $\left(0^{\circ}\right.$ line $)$. The $0^{\circ}$ line along the forebody surface experiences the lowest pressures. The experimental $90^{\circ}$ and $270^{\circ}$ values, as well as the $120^{\circ}$ and $240^{\circ}$ values again line up fairly well. Note that the static pressure distribution for the $\alpha=0^{\circ}$ case (Fig. 3(a)) would lie approximately centered in between the $\alpha=3^{\circ}$ distributions presented for the $0^{\circ}$ to $180^{\circ}$ circumferential range in Fig. 3(b).

In general, the trends at the other Mach numbers investigated are the same as for Mach 2.5, though the Mach 3.0 and 3.5 cases both showed several discrepancies. As is seen in Fig. 4(a), the Mach 3.0 solution showed the experimental normalized static pressure distribution consistently offset below the CFD solution. As is seen in Fig. 4(b), the Mach 3.5 static pressure distributions showed good agreement at the front of the forebody, but the experimental static pressures were found to be lower than the CFD static pressure distribution line further downstream. The source of the discrepancies could be attributed to increases in velocities in the SWT not accounted for in the CFD. Axial (in addition to planar) wind tunnel gradients on the order of $\triangle \mathrm{Mach} 0.04$ spanning over the volume occupied by the model were found in the Mach 3.0 and 3.5 calibration curves (at high Re\#/ft). This is important since all CFD cases were run exactly at 2.00, 2.50, 3.00 and 3.50. As will be discussed in the boundary-layer rake section, even the small Mach changes mentioned above could help account for the discrepancies found in the results.

Normalized Flowfield Rake Pitot Pressure Contour Plots at $x=98.95$ in. $\left(\theta=5^{\circ}\right.$ to $35^{\circ}, 175^{\circ}$ to $\left.145^{\circ}\right)$

A comparison of CFD and experimental flowfield rake data obtained at $\mathrm{x}=98.95$ in. is now made. Located near a plane slightly aft of the centerbody tips, flowfield rakes were used to measure the variation of the pitot pressure circumferentially on the leeward and windward sides of the forebody. It should be noted that experimentally there were only four rakes $\left(\theta=5^{\circ}, 15^{\circ}\right.$, $25^{\circ}$ and $35^{\circ}$ ) used for each run, but the model was run at both positive and negative angles-of-attack. This allowed the data to be compiled as shown in Fig. 5 using data from eight rakes (adding data at $\theta=145^{\circ}$, $155^{\circ}, 165^{\circ}$ and $175^{\circ}$ ). Figure $1(\mathrm{~b})$ gives a general idea of where these eight rakes (at $\mathrm{x}=98.95 \mathrm{in}$.) are located. The variable plotted in Fig. 5 is the normalized pitot pressure $\left(\mathrm{P}_{\text {pitot }} / \mathrm{P}_{\text {tunnel total }}\right)$. The CFD solution used in the comparison is that obtained assuming a turbulent boundary-layer over the entire forebody. The two lefthand side contour quadrants (top and bottom) represent the experimental data, and they can be directly compared with the CFD results that have been placed to the right-hand side. Refer to the visual aids to the right of the quadrant plots to better understand the data being presented. The surface of the forebody has been depicted in each quadrant figure with a dashed line.

The particular cases shown in Fig. 5 are the Mach 2.5 comparison for $\alpha=3^{\circ}$ (Fig. 5(a)) and the Mach 3.5 comparison for $\alpha=3^{\circ}$ (Fig. 5(b)). Overall, comparing CFD to experiment, the agreement is very good for all the conditions tested. The bottom (windward) rakes show higher gradients closer to the forebody surface as compared to the top (leeward) rakes, as may be expected when at an angle-of-attack. WIND's PNS capability is seen to be capturing the general features of the flowfield quite well. The trends at the other Mach numbers are very similar to those just discussed. Generally, the windward side experienced slightly higher pitot pressures than the leeward in the outer "freestream" region of the external forebody flow.

\section{Temperature-Sensitive Paint (TSP) Data.}

Figure 6(a) shows experimental TSP data for each of the four Mach number cases at $\alpha=0^{\circ}$ (without grit application). The equilibrium surface temperature can be inferred by the TSP surface profile distributions. This plot can help characterize the transition from a laminar to a turbulent boundary-layer occurring along the forebody. For Mach 2.0, 2.5 and 3.0, similar temperature distributions are found where the minimum-maximum-minimum peaks are occurring at the same axial locations along the forebody. The general location for this "transition" region is thus occurring from $\mathrm{x} \sim 20$ to $\sim 35$ in. The sudden increase in temperature is known to correspond to the beginning of transition. ${ }^{7}$ Though the Mach 3.5 curve differs from the other three, it appears the peak occurs slightly aft of the peak for the other Mach numbers. Taken as a whole, however, this data shows transition is occurring at roughly the same location, which may be expected since the Re\#/ft is approxi-mately the same for all four cases.

Figure 6(b) shows the same four flow conditions, but now the grit has been applied at $x=3.0$ in. This is evident from the "blip" in the temperature distribution seen at this location. Comparing with and without grit 
application, the temperature distributions have changed. Both Mach 2.0 and 2.5 (with grit applied) show approximately a constant temperature level that is near that seen on the aft portion of the forebody in the no grit applied case (indicating the grit has tripped the boundary-layer to turbulent further upstream). Though Mach 3.0 and 3.5 with grit differ, transition to turbulent is seen to have occurred further upstream than in the no grit cases. This experimental TSP data (along with the experimental boundary-layer rake data) will be compared with the turbulent and laminar CFD results in the next section to investigate this transition further.

Normalized Boundary-Layer Rake Pitot Pressure Profiles

In the experiment, four boundary-layer rakes were placed in various positions axially and circumferentially along the forebody surface. For each test condition, the model was run at both a positive and negative angle-ofattack. Therefore, experimental data can be reduced to eight rake locations using symmetry considerations and positive/negative angle-of-attack information (see Fig. 1(b)). The rake locations through symmetry considerations reduce to the following: ( $x=32.5$ in., $\left.\theta=58^{\circ}, 122^{\circ}\right),\left(x=53.94\right.$ in., $\left.\theta=9^{\circ}, 171^{\circ}\right),(x=75.38$ in., $\left.\theta=87^{\circ}, 93^{\circ}\right)$, and $\left(x=100.33\right.$ in., $\left.\theta=10^{\circ}, 170^{\circ}\right)$. The distance normal to the surface versus the normalized pitot pressure $\left(\mathrm{P}_{\text {pitot }} / \mathrm{P}_{\text {tunnel total }}\right)$ will be plotted. Turbulent and laminar sharp-nose grid CFD solutions have been obtained for all the cases investigated and are compared with experimental results in this section. Plotting both CFD solutions together with the experimental data allows for a "first order" estimate of the type of boundary-layer (laminar, transitional, or turbulent) over the entire forebody. Though this is not quite accurate (since the downstream boundary-layer is dependent on what the boundary-layer characteristic has been upstream), the comparison will be shown to be good enough for the purposes of this study and provides "bounds" on the type of flow.

Figure 7 shows the four boundary-layer rakes for the Mach 2.0 case at $\alpha=0^{\circ}$, with additional experimental data included for the case where grit was applied to the sharp-nose forebody configuration to trip the boundary-layer to turbulent. Over the forebody, the experimental boundary-layer data without grit applied shows good agreement with the turbulent CFD data at the three downstream rake locations. However, the boundary-layer rake located at $\mathrm{x}=32.5 \mathrm{in}$. shows the data does not entirely lie on the turbulent solution profile, but rather has a slightly thinner profile. This agrees with the no grit TSP results presented previously, which showed that the turbulent boundarylayer was not obtained until somewhere near this rake position.

The assumption made in the flowfield rake section discussed previously (in regards to presenting the experimental flowfield rake data at $\mathrm{x}=98.95 \mathrm{in}$. with only the turbulent CFD data) is quite sufficient for sharp-nose forebody comparisons. Note that laminar and turbulent CFD static pressure distributions were very identical. Hence, this was the reason for only plotting experimental static pressure data with the turbulent CFD data.

Comparing the Mach 2.0 results with the gritapplied experimental data, it can be seen in Figs. 7(a-d) that the profiles lie almost on top of the turbulent CFD solution profiles. The profiles are seen to be slightly thicker than profiles without grit. Transition has occurred further upstream than the case without grit. Therefore, agreement with the turbulent CFD profile is much better for the case with grit.

It should also be noted, though not presented here, that all eight experimental rake profiles for the $\alpha=3^{\circ}$ case (without grit) lie on top of their respective turbulent CFD profiles. This is the case for all cases tested with the sharp-nose grid. Therefore, there is very good experimental and CFD agreement at angle-ofattack for the sharp-nose forebody, and WIND (with all the assumptions made) has done a good job of capturing the pertinent flow phenomenon. This suggests that transition from laminar to turbulent occurs further upstream when at angle-of-attack than is seen for the $\alpha=0^{\circ}$. As would be expected at an angle-of-attack, the leeward side rakes, especially those closest to the top of the forebody ( $x=53.94$ and 100.33 in.), display a thicker boundary-layer profile than the windward side rakes at the same axial locations. In addition, as the flow proceeds downstream over the forebody, the effects of expansion can be seen to be taking place, with "freestream" pitot pressures continually decreasing. Also note that the pitot pressure does not reach a constant value outside the "boundary-layer" in these plots because of the "conical" nature of the flow.

Mach 2.5 and 3.0 sharp-nose forebody experimental data showed very similar trends to the Mach 2.0, $\alpha=0^{\circ}$ and $3^{\circ}$ results just discussed (i.e., experiment and turbulent CFD pitot pressure profiles don't line up exactly until after $x=32.5$ in. for $\alpha=0^{\circ}$, and they line up at all locations measured for $\alpha=3^{\circ}$ ). However, Mach 3.5 actually showed that the experimental boundary-layer data without grit does not line up with the turbulent CFD profile until somewhere after $x=53.94$ in. Transition appears to have occurred further back as compared to the other Mach number cases (as might be ascertained from the TSP data in Fig. 6(a)).

Mach 3.5 pitot pressures in the "freestream" (for the experiment and CFD) were offset from one another at various axial stations. Higher "freestream" pitot pressures were obtained for the experimental data as compared to the CFD data at upstream locations, while lower "freestream" experi-mental pitot pressures were obtained at the downstream locations. The trend agreed with 10 - by 10 -foot SWT data showing an increasing freestream Mach number with axial travel downstream in the tunnel for certain conditions (and therefore a decreasing "freestream" pitot pressure). Several Mach 
number perturbation CFD runs (on the order $\triangle \mathrm{Mach}$ $\sim 0.04$ ) showed the discrep-ancies between experimental and CFD boundary-layer rake "freestream" values could be explained by these slight Mach number gradients.

\section{Blunt-nose Results}

Numerous FNS runs of the WIND code have been completed for the blunt-nose Medium grid (every other grid point) at $\alpha=0^{\circ}$ and at $\alpha=3^{\circ}(M \cong 2.0,2.5,3.0$, and 3.5). A summary of the results for these cases follows.

Comparing boundary-layer profiles, trends at Mach 2.0 and Mach 2.5 (for $\alpha=0^{\circ}$ ) are somewhat similar to the sharp-nose Mach 2.0, 2.5 and $3.0\left(\alpha=0^{\circ}\right)$ forebody trends. Figures 8(a-d) shows the Mach 2.5, $\alpha=0^{\circ}$, bluntnose boundary-layer rake profile comparisons (all without grit) over the forebody length. At $x=32.5$ in., the experimental data actually is seen to lie closer to a laminar boundary-layer profile than a turbulent one. However, further downstream at $x=53.94$ in., the experimental profiles are lying on top of the turbulent CFD solution profiles. Generally speaking for these cases just mentioned, the thickness for both the sharpand blunt-nose profiles, as well as the normalized "freestream" pitot pressure values, agree quite well with each other (station-to-station) over the forebody surface.

Mach 3.0 and 3.5 boundary-layer rake results, however, show that the experimental data (no grit was applied in any of the blunt-nose cases) do not lie directly on the turbulent CFD solution until further downstream than had been seen in the sharp-nose forebody solutions. For the Mach 3.5, $\alpha=0^{\circ}$ solution, the experimental profile lies atop the laminar CFD profile even up to $\mathrm{x}=53.94$ in. Therefore, transition is occurring downstream of this station for the blunt-nose condition, whereas this type of definitive statement could not be made for the corresponding sharp-nose cases (where the upstream experiment profiles were found only slightly offset from the turbulent CFD solutions).

For $\alpha=3^{\circ}$, the trends are actually slightly different for several of the blunt-nose cases as compared to the sharp-nose cases. As before for the sharp-nose cases at Mach 2.0 and 2.5, the experimental profiles lay nearly atop the turbulent CFD profiles at all the rake locations. However, the Mach 3.0 and 3.5 experimental profiles (up to and including $\mathrm{x}=53.94 \mathrm{in}$. on the windward side) lie either somewhere in between turbulent and laminar CFD profiles or directly on top of the laminar profile (see Fig. 9 for Mach 3.0). This was not seen in the sharp-nose cases at any Mach number when the forebody was at any angle-of-attack.

In summary, it may be deduced that, in general, transition has occurred at axial locations ahead of where the experimental profile finally matches the turbulent solution. When taking all boundary-layer rakes as a whole, this overall view of the data can be compared to the experimental TSP data (as was shown in Fig. 6). The conclusions drawn from the CFD-combined experimental normalized pitot pressure plots show good general agreement with the TSP data conclusions, and provide some general idea of boundary-layer transition location and characteristics. Overall, the PNS capability is seen to have done a good job in returning worthwhile results that agree well with experiment.

\section{Summary And Conclusion}

In comparisons involving NASA Glenn's 10- by 10-foot Supersonic Wind Tunnel experimental results, very good agreement between WIND Code CFD and experiment has been found. Using WIND's PNS capability where possible, the coarser grids, and the Baldwin-Lomax algebraic turbulence model allowed for great reductions in the computational time while still maintaining good agreement with the experimental results. Running CFD concurrently with experimental testing allowed for one's information to feed directly into the planning for the other. Future use of the CFD models to obtain reasonably accurate information that was not obtained with the experiment is now an option. The WIND code has been found to be a good solver for the applications set forth in this study.

\section{Grid Convergence Study}

\section{Appendix}

A grid convergence study for the sharp-nose and blunt-nose grids has been performed based on the procedures outlined in Ref. 6 . The total drag coefficient (pressure plus viscous drag coefficient) was the parameter used for comparison in these studies.

First, for the sharp-nose grid, the Mach $2.5\left(\alpha=0^{\circ}\right)$ flowfield was computed on three grids, each with twice the number of grid points in the $i-, j-$, and $k$-directions as the previous grid (i.e., Coarse, Medium and Fine grid spacing). The Fine grid was chosen as the reference grid and is referred to as grid 1, the Medium grid is referred to as grid 2, and the Coarse as grid 3.

The Grid Convergence Index (GCI), similar to the Richardson error estimator and based on the generalized theory of Richardson Extrapolation, provides a calculated error band on the solution functionals (total drag coefficient in this case). It also provides a measure of uncertainty for grid convergence. Though two levels of sequencing (three grids) were utilized with a constant grid refinement ratio of 2, the GCI was calculated using a Factor of safety $\left(\mathrm{F}_{\mathrm{s}}\right)$ equal to 3 in order to be conservative. Table 1 presents the sharp-nose grid's relevant information for the grid convergence study. Defining " $p$ " (the order of convergence) per Ref. 6 , it is calculated by using the solution functionals obtained from the three grids and the grid refinement ratio. For the sharp-nose grid, using Table 1 values, $p$ was calculated as $\sim 1.74$. This shows the solution is not quite at a second-order level of convergence. This may be expected since, with the PNS 
capability, WIND automatically changes to a first-order explicit operator in the $i$-direction (marching direction) only, while keeping second-order operators in the other directions. The Fine grid GCI $\left(\mathrm{GCI}_{12}\right)$ was calculated as 0.05 percent, while the Medium grid GCI $\left(\mathrm{GCI}_{23}\right)$ was calculated as 0.17 percent. The solutions were found to be in the asymptotic range $\left[\mathrm{GCI}_{23} /\left(\mathrm{GCI}_{12} * 2^{\mathrm{p}}\right) \approx 1.00\right]$. These values were deemed good enough for the purposes of this study. The Medium grid has been used throughout this paper for the sharp-nose forebody cases.

For the blunt-nose grid, the Mach $2.5\left(\alpha=0^{\circ}\right)$ flowfield was also computed on three grids, each with twice the number of grid points in the $i-, j-$, and $k$-directions as the previous grid (i.e., Coarse, Medium and Fine grid spacing). Using the same procedure as described above, Table 2 presents the relevant bluntnose grid information for the grid convergence study. The order of convergence $p$ was calculated as $\sim 1.52$. This shows that the solution is not at a second-order level of convergence, where FNS calculations for the blunt-nose case use second-order explicit operators in all directions. The difference between theoretical and calculated order of convergence may be due to grid stretching, grid quality, nonlinearities in the solutions (such as the presence of shocks) and possibly other factors. ${ }^{6}$ The Fine grid GCI $\left(\mathrm{GCI}_{12}\right)$ was calculated as 0.38 percent, while the Medium grid GCI $\left(\mathrm{GCI}_{23}\right)$ was calculated as 1.09 percent. The solutions were found to be in the asymptotic range $\left[\left(\mathrm{GCI}_{23} /\left(\mathrm{GCI}_{12} * 2^{\mathrm{p}}\right)\right) \approx 1.00\right]$. These values were deemed good enough for the purposes of this study. The Medium grid has been used throughout this paper for the blunt-nose forebody cases.

\section{$\underline{\text { References }}$}

${ }^{1}$ Trefny, C.J., An Air-Breathing Launch Vehicle Concept for Single-Stage-to-Orbit, NASA TM-1999-209089, 1999.

${ }^{2}$ Kim, H.D., and Frate, F.C., "Experimental Evaluation of the Effect of Angle-of-Attack on the External Aerodynamics and Mass Capture of a Symmetric, Three-Engine Air-Breathing Launch Vehicle Configuration at Supersonic Speeds," 37th JANNAF Combustion Subcommittee, 25th Airbreathing Propulsion Sub-committee, and 19th Propulsion Systems Hazards Subcommittee Joint Meeting, Nov 13-17, 2000.

${ }^{3}$ Seddon, J., and Goldsmith, E.L., Practical Intake Aerodynamic Design, Anderson, B.H. and Towne, C.E., Application of Computational Fluid Dynamics to Inlets, AIAA Education Series, 1993.

${ }^{4}$ Akdag, V. and Wulf, A., "Integrated Geometry and Grid Generation System for Complex Configurations," Software Systems for Surface Modeling and Grid Generation, NASA CP-3143, Apr. 1992.

${ }^{5}$ WIND Documentation Home Page, http://www.grc.nasa.gov/www/winddocs/index.html, The WIND Code, WWW page created by Charles Towne, Version 3.0, Accessed Jan.- Dec. 2000.

${ }^{6}$ Roache, P.J., Verification and Validation in Computational Science and Engineering, Hermosa Publishers, Albuquerque, New Mexico, 1998.

${ }^{7}$ Schlichting, H., Boundary-Layer Theory, Sixth Edition, McGraw-Hill, New York, 1968.

Table 1.-Sharp-nose Forebody Grid Convergence Study Results - Mach 2.5, $\alpha=0^{\circ}$

\begin{tabular}{|l|c|c|c|c|}
\hline \multicolumn{1}{|c|}{ Grid Number } & $\begin{array}{c}\text { Grid Spacing } \\
\text { (Normalized by } \\
\text { finest grid spacing) }\end{array}$ & $\begin{array}{c}\text { Total CPU time } \\
\text { (Corrected for } \\
\text { machine use) }\end{array}$ & $\begin{array}{c}\text { Total Drag } \\
\text { Coefficient }\end{array}$ & $\begin{array}{c}\text { Calculated order } \\
\text { of convergence } \\
(p)\end{array}$ \\
\cline { 1 - 4 } Fine (1) - 000 sequencing in WIND & 1 & $\sim 75,500 \mathrm{sec}$ & 0.108639 & \multirow{2}{*}{1.74} \\
\hline Medium (2) -111 sequencing in WIND & 2 & $\sim 3,000 \mathrm{sec}$ & 0.108596 & \\
\hline Coarse (3) -222 sequencing in WIND & 4 & $\sim 200 \mathrm{sec}$ & 0.108452 & \\
\hline
\end{tabular}

Table 2.-Blunt-nose Forebody Grid Convergence Study Results - Mach 2.5, $\alpha=0^{\circ}$.

\begin{tabular}{|l|c|c|c|c|}
\hline \multicolumn{1}{|c|}{ Grid Number } & $\begin{array}{c}\text { Grid Spacing } \\
\text { (Normalized by } \\
\text { finest grid spacing) }\end{array}$ & $\begin{array}{c}\text { Total CPU time } \\
\text { (Corrected for } \\
\text { machine use) }\end{array}$ & $\begin{array}{c}\text { Total Drag } \\
\text { Coefficient }\end{array}$ & $\begin{array}{c}\text { Calculated order } \\
\text { of convergence } \\
(p)\end{array}$ \\
\hline Fine (1) -000 sequencing in WIND & 1 & $\sim 446,500 \mathrm{sec}$ & 0.107664 & \multirow{2}{*}{1.52} \\
\hline Medium $(2)-111$ sequencing in WIND & 2 & $\sim 17,000 \mathrm{sec}$ & 0.107411 \\
\cline { 1 - 3 } Coarse (3)-222 sequencing in WIND & 4 & $\sim 2,000 \mathrm{sec}$ & 0.106686 & \\
\hline
\end{tabular}




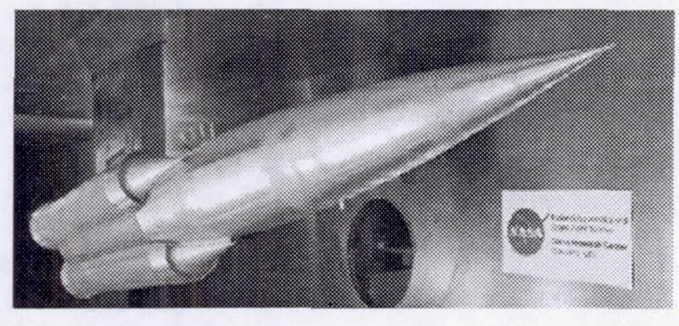

(a)

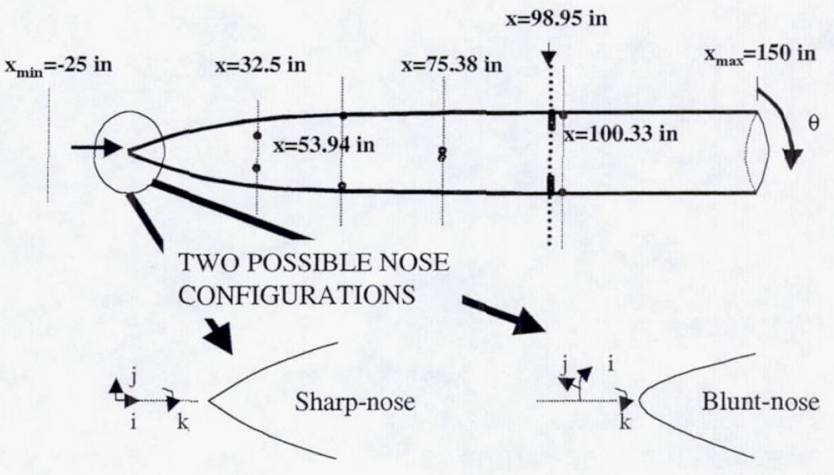

(b)

Figure 1 - (a) Scaled-down GTX Model, (b) Schematic and general description of forebody used for CFD.

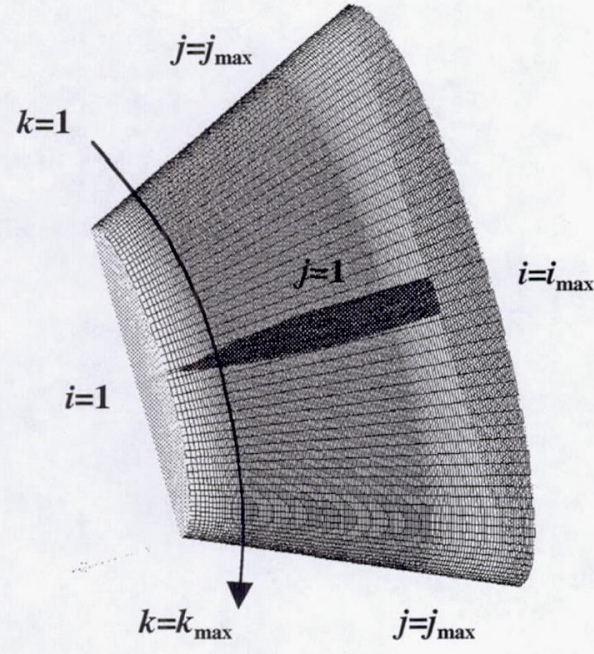

(a)

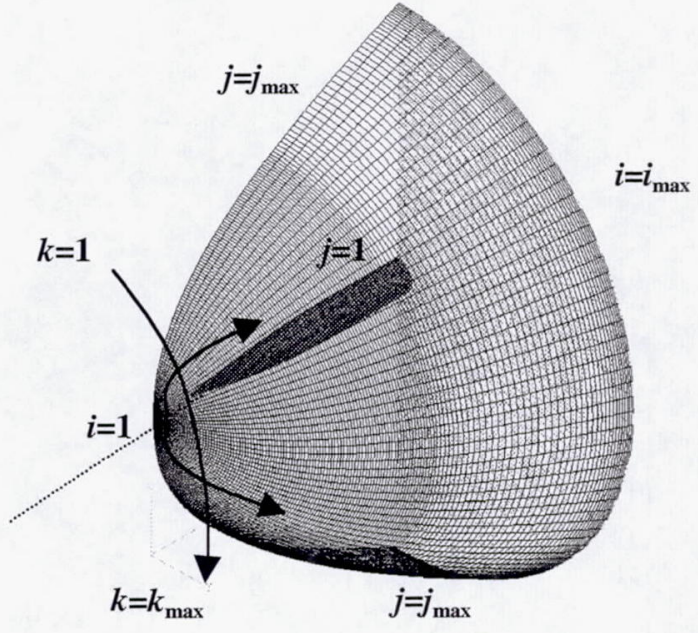

(b)

Figure 2 - Isometric views, (a) Sharp-nose forebody grid, (b) Blunt-nose forebody grid. (Every grid point for the finest grid is shown.) 


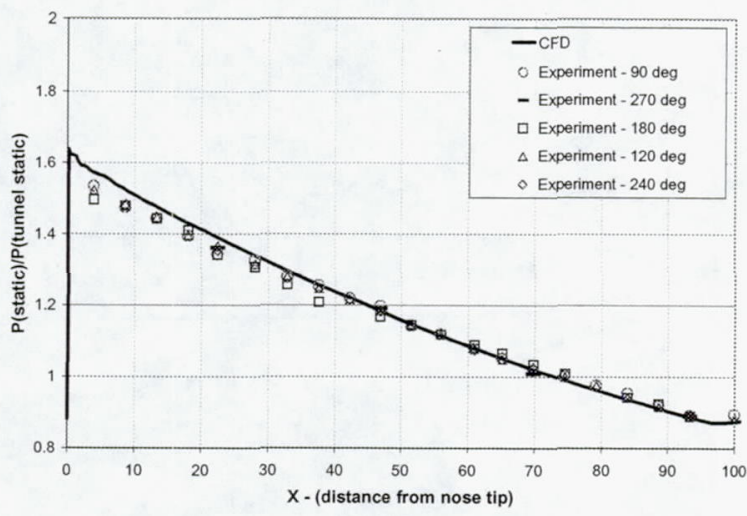

(a)

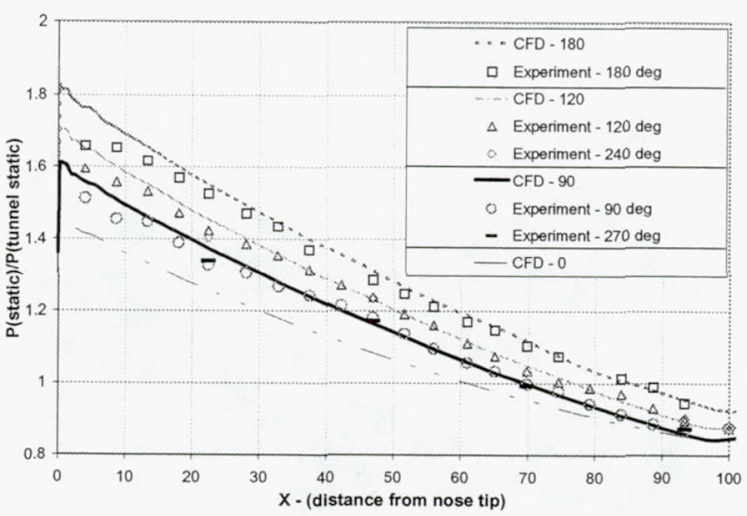

(b)

Figure 3 - Sharp-nose Forebody Normalized Static Pressure Distribution, (a) Mach 2.5, $\alpha=0^{\circ}$,

(b) Mach 2.5, $\alpha=3^{\circ}$.

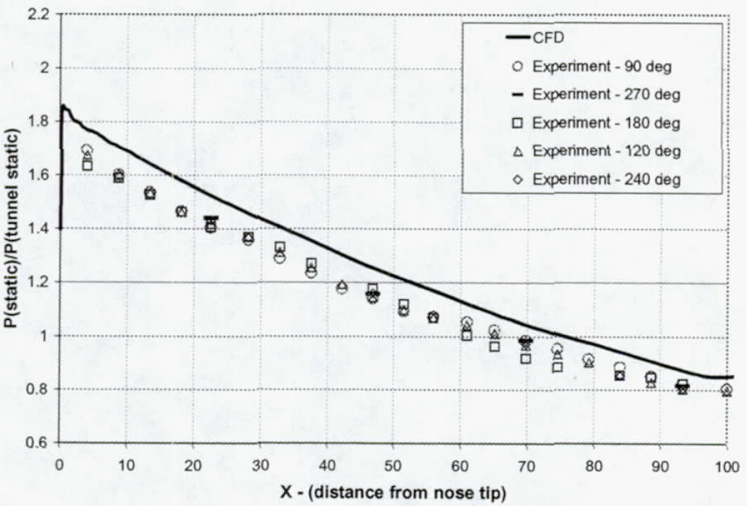

(a)

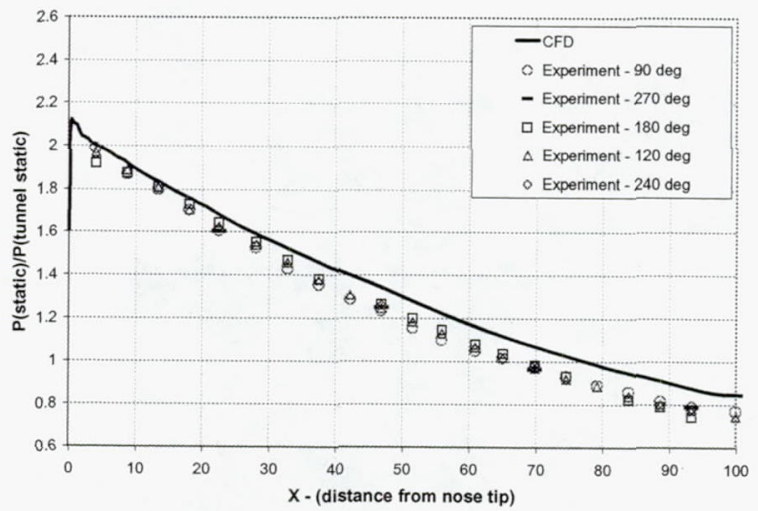

(b)

Figure 4 - Sharp-nose Forebody Normalized Static Pressure Distribution, (a) Mach 3.0, $\alpha=0^{\circ}$,

(b) Mach 3.5, $\alpha=0^{\circ}$. 

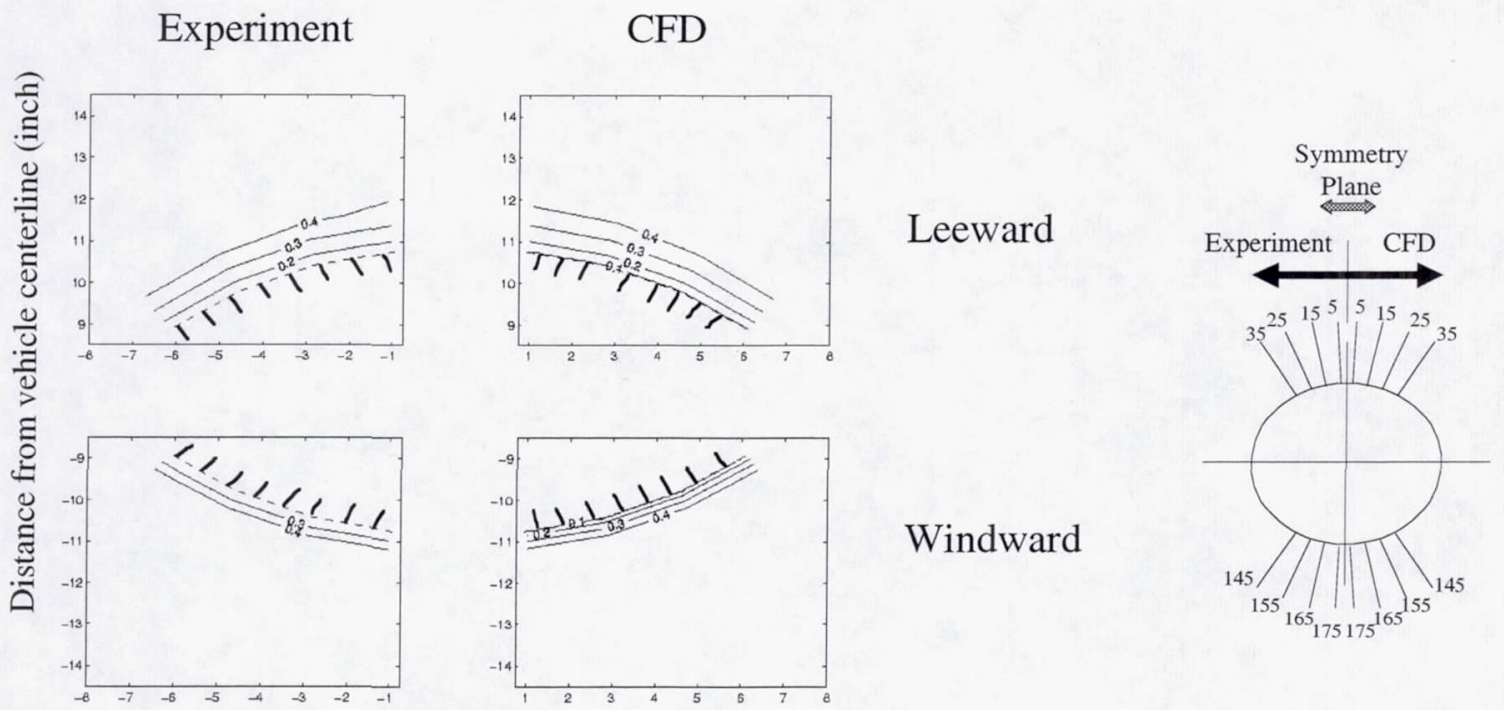

Distance from vehicle centerline (inch)

(a)
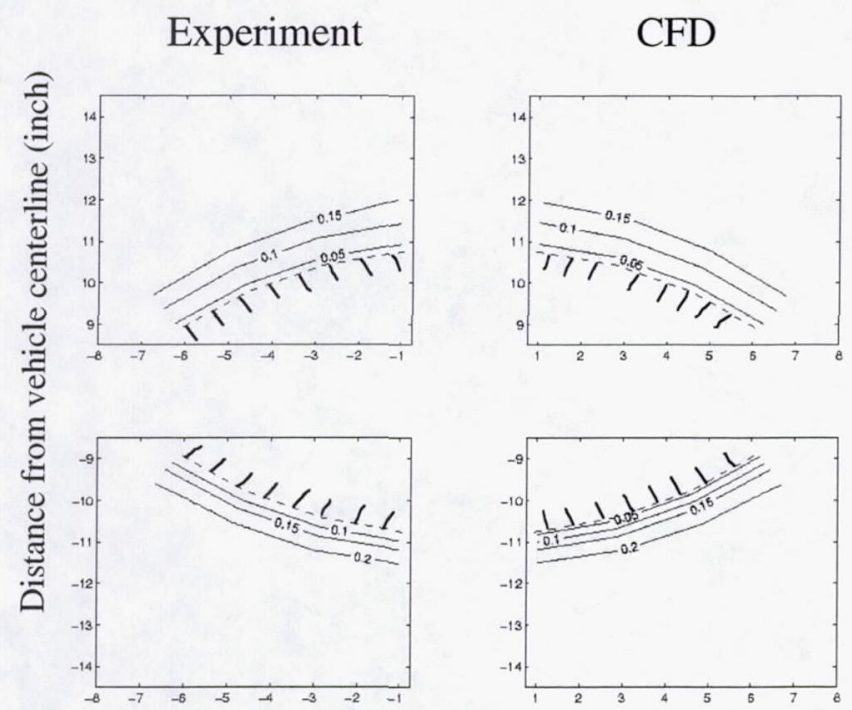

Leeward

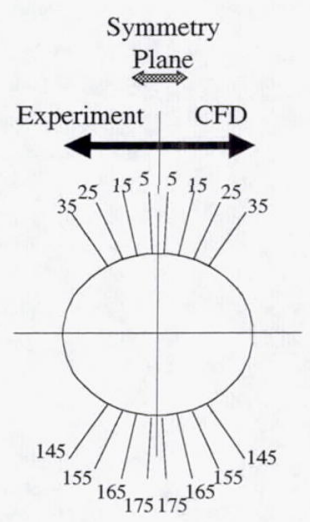

Distance from vehicle centerline (inch)

(b)

Figure 5 - Sharp-nose Flowfield Rake Normalized Pitot Pressure Contour Plots for $\alpha=3^{\circ}$ at $x=98.95$ inches (experiment vs. turbulent CFD),
(a) Mach 2.5, $\alpha=3^{\circ}$,
(b) Mach 3.5, $\alpha=3^{\circ}$. 


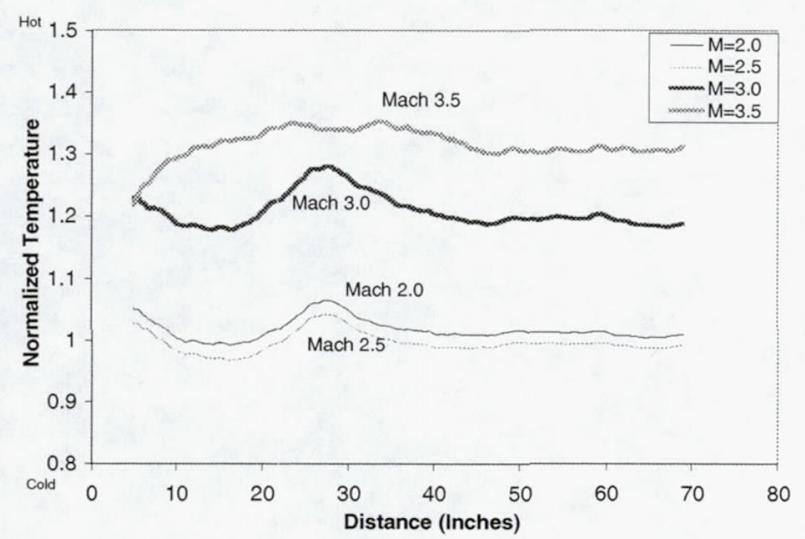

(a)

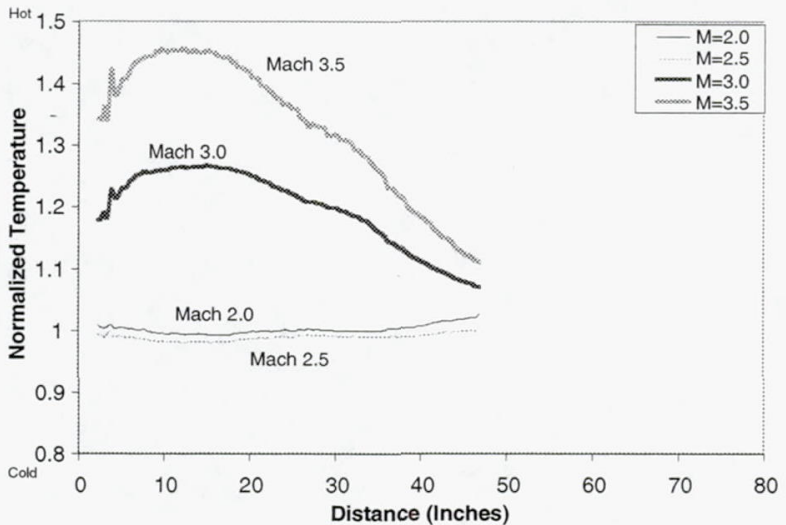

(b)

Figure 6 - Sharp-nose normalized temperature distribution over part of forebody for $\alpha=0^{\circ}$ cases,

(a) No grit applied, (b) With grit applied at $\mathrm{x}=3.0$ inches.

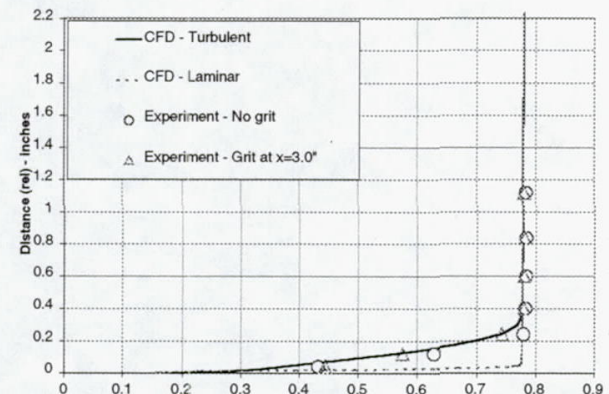

(a)

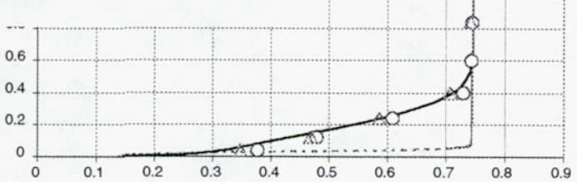

(b)

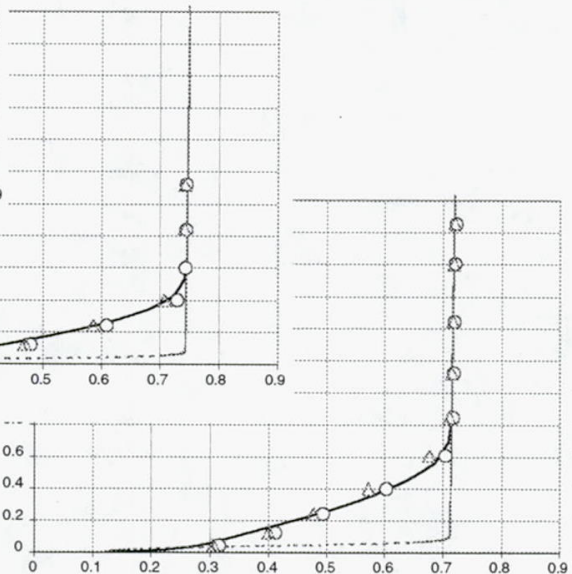

(c)
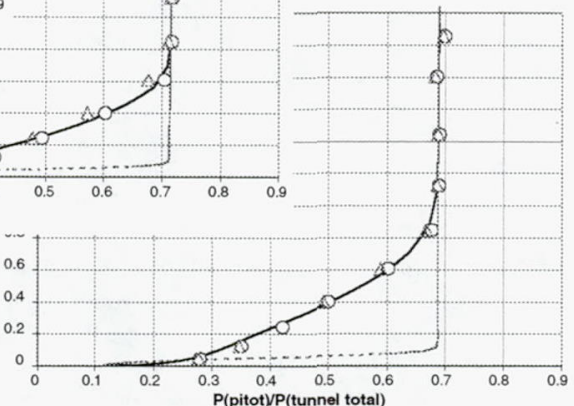

(d)

Figure 7 (a-d) - Sharp-nose Boundary-Layer Rake Normalized Pitot Pressure Profiles along the forebody at various axial stations (turbulent and laminar CFD vs. experimental (with and without grit)),

Mach 2.0, $\alpha=0^{\circ}$,

(a) $x=32.5$ inches, (b) $x=53.94$ inches, (c) $x=75.38$ inches, (d) $x=100.33$ inches. 
(a)

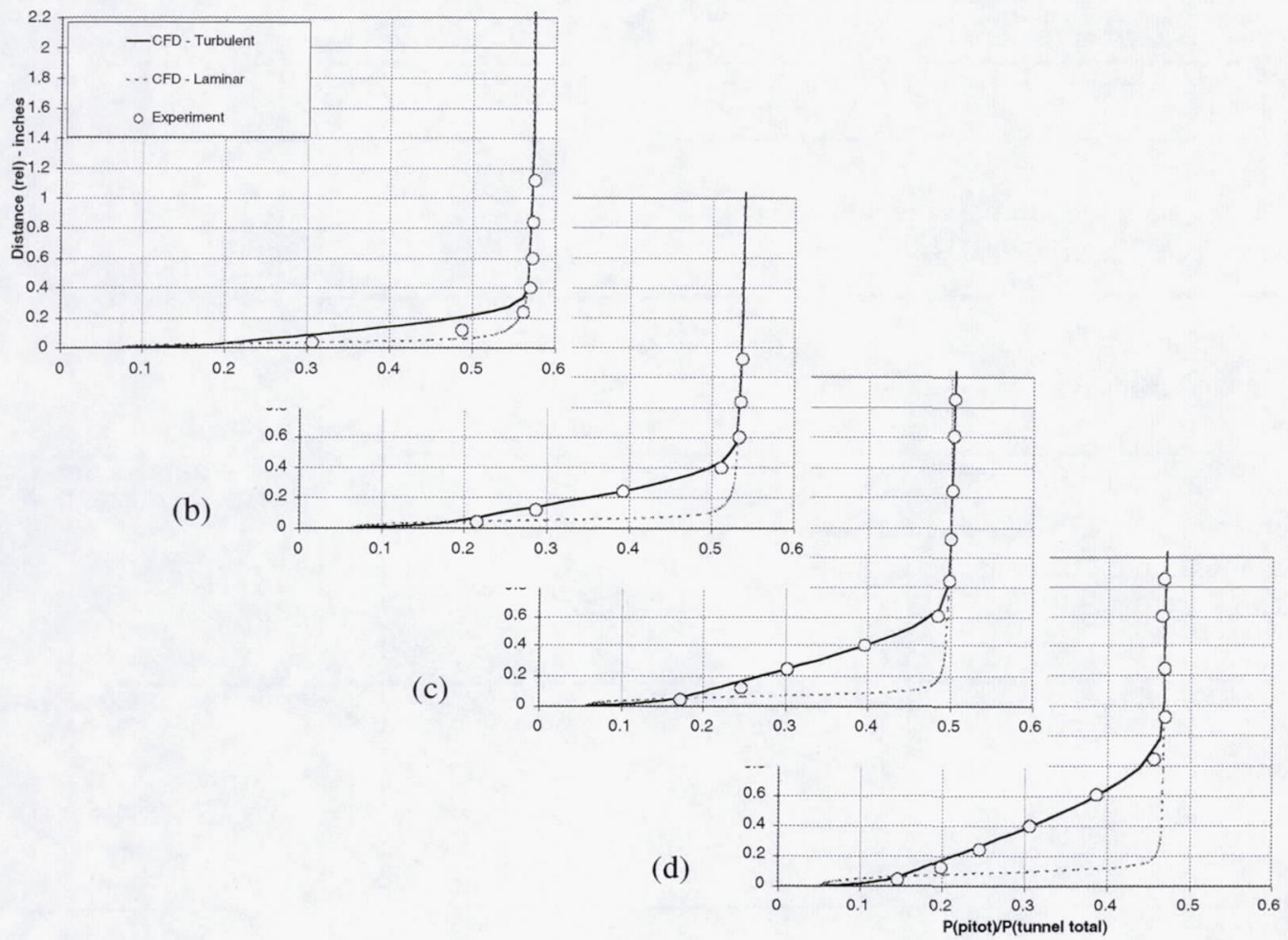

Figure 8 (a-d) - Blunt-nose Boundary-Layer Rake Normalized Pitot Pressure Profiles along the forebody at various axial stations (turbulent and laminar CFD vs. experimental (all without grit)), Mach 2.5, $\alpha=0^{\circ}$, (a) $x=32.5$ inches, (b) $x=53.94$ inches, (c) $x=75.38$ inches, (d) $x=100.33$ inches.

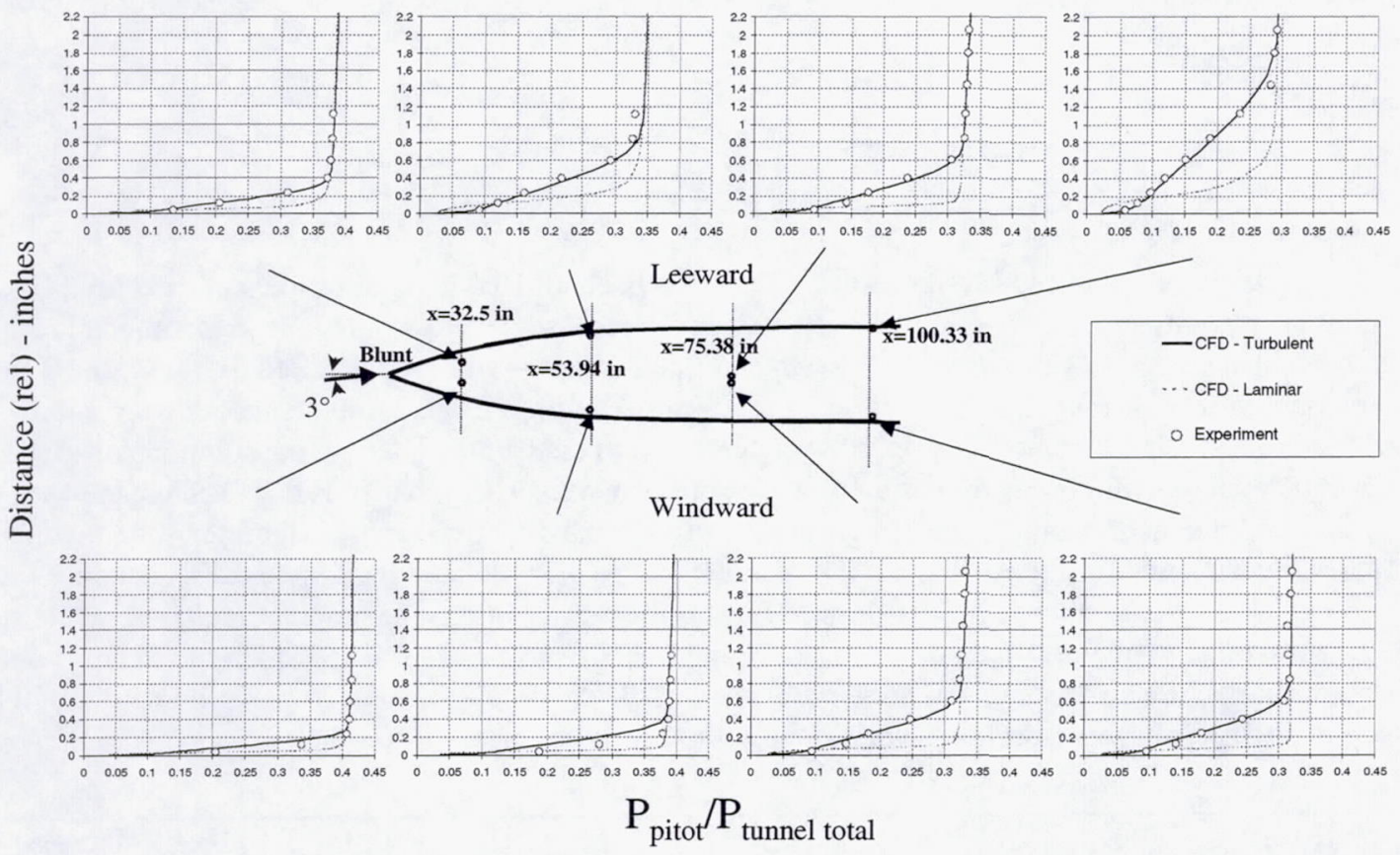

Figure 9 - Blunt-nose Boundary-Layer Rake Normalized Pitot Pressure Profiles along the forebody at various axial stations, Mach 3.0, $\alpha=3^{\circ}$. 
Public reporting burden for this collection of information is estimated to average 1 hour per response, including the time for reviewing instructions, searching existing data sources, gathering and maintaining the data needed, and completing and reviewing the collection of information. Send comments regarding this burden estimate or any other aspect of this collection of information, including suggestions for reducing this burden, to Washington Headquarters Services, Directorate for Information Operations and Reports, 1215 Jefferson Davis Highway, Suite 1204, Arlington, VA 22202-4302, and to the Office of Management and Budget, Paperwork Reduction Project (0704-0188), Washington, DC 20503.

\begin{tabular}{|l|l|l|}
\hline 1. AGENCY USE ONLY (Leave blank) & 2. REPORT DATE & 3. REPORT TYPE AND DATES COVERED
\end{tabular}

May 2001

Technical Memorandum

\section{TITLE AND SUBTITLE}

5. FUNDING NUMBERS

Wind Code Application to External Forebody Flowfields

With Comparisons to Experimental Results

6. AUTHOR(S)

F.C. Frate and H.D. Kim

\section{PERFORMING ORGANIZATION NAME(S) AND ADDRESS(ES)}

National Aeronautics and Space Administration

John H. Glenn Research Center at Lewis Field

Cleveland, Ohio 44135-3191

WU-708-73-20-00

\section{SPONSORING/MONITORING AGENCY NAME(S) AND ADDRESS(ES)}

National Aeronautics and Space Administration

Washington, DC 20546-0001

8. PERFORMING ORGANIZATION REPORT NUMBER

E-12532

\section{SUPPLEMENTARY NOTES}

Prepared for the 39th Aerospace Sciences Meeting and Exhibit sponsored by the American Institute of Aeronautics and Astronautics, Reno, Nevada, January 8-11, 2001. F.C. Frate, Dynacs Engineering Company, Inc., 2001 Aerospace Parkway, Brook Park, Ohio 44142; and H.D. Kim, NASA Glenn Research Center. Responsible person, Hyun Dae Kim, organization code $5850,216-433-8344$.

12a. DISTRIBUTION/AVAILABILITY STATEMENT 12b. DISTRIBUTION CODE

Unclassified - Unlimited

Subject Category: 02

Distribution: Nonstandard

Available electronically at http://gltrs. grc.nasa.gov/GLTRS

This publication is available from the NASA Center for AeroSpace Information, 301-621-0390.

13. ABSTRACT (Maximum 200 words)

The WIND Code, a general purpose Navier-Stokes solver, has been utilized to obtain supersonic external flowfield CFD solutions over an axisymmetric, parabolic forebody with comparisons made to wind tunnel experimental results. Various cases have been investigated at supersonic freestream conditions ranging from Mach 2.0 to 3.5 , at $0^{\circ}$ and $3^{\circ}$ angles-ofattack, and with either a sharp-nose or blunt-nose forebody configuration. Both a turbulent (Baldwin-Lomax algebraic turbulence model) and a laminar model have been implemented in the CFD. Obtaining the solutions involved utilizing either the parabolized- or full-Navier-Stokes analyses supplied in WIND. Comparisons have been made with static pressure measurements, with boundary-layer rake and flowfield rake pitot pressure measurements, and with temperaturesensitive paint experimental results. Using WIND's parabolized Navier-Stokes capability, grid sequencing, and the Baldwin-Lomax algebraic turbulence model allowed for significant reductions in computational time while still providing good agreement with experiment. Given that CFD and experiment compare well, WIND is found to be a good computational platform for solving this type of forebody problem, and the grids developed in conjunction with it will be used in the future to investigate varying freestream conditions not tested experimentally.

\section{SUBJECT TERMS}

Computational fluid dynamics; Supersonic boundary layers; Forebodies; Boundary layer transition

\begin{tabular}{|c|c|}
\hline $\begin{array}{c}\text { 17. SECURITY CLASSIFICATION } \\
\text { OF REPORT }\end{array}$ & $\begin{array}{c}\text { 18. SECURITY CLASSIFICATION } \\
\text { OF THIS PAGE } \\
\text { Unclassified }\end{array}$ \\
Unclassified
\end{tabular}
19. SECURITY CLASSIFICATION OF ABSTRACT Unclassified

15. NUMBER OF PAGES

\section{7}

16. PRICE CODE

20. LIMITATION OF ABSTRACT 